\title{
GRAPH MATCHING FOR THE REGISTRATION OF PERSISTENT SCATTERERS TO OPTICAL OBLIQUE IMAGERY
}

\author{
L. Schack ${ }^{\mathrm{a} *}$ U. Soergel ${ }^{\mathrm{b}}$, C. Heipke ${ }^{\mathrm{a}}$ \\ ${ }^{\text {a }}$ Institute of Photogrammetry and GeoInformation, Leibniz Universität Hannover, Germany - \\ (schack, heipke)@ipi.uni-hannover.de \\ ${ }^{\mathrm{b}}$ Institute for Photogrammetry, Universität Stuttgart, Germany - \\ uwe.soergel@ifp.uni-stuttgart.de
}

Commission VII, WG VII/6

KEY WORDS: SAR, Matching, Registration, optical, imagery

\begin{abstract}
:
Matching Persistent Scatterers (PS) to airborne optical imagery is one possibility to augment applications and deepen the understanding of SAR processing and products. While recently this data registration task was done with PS and optical nadir images the alternatively available optical oblique imagery is mostly neglected. Yet, the sensing geometry of oblique images is very similar in terms of viewing direction with respect to SAR. We exploit the additional information coming with these optical sensors to assign individual PS to single parts of buildings. The key idea is to incorporate topology information which is derived by grouping regularly aligned PS at facades and use it together with a geometry based measure in order to establish a consistent and meaningful matching result. We formulate this task as an optimization problem and derive a graph matching based algorithm with guaranteed convergence in order to solve it. Two exemplary case studies show the plausibility of the presented approach.
\end{abstract}

\section{INTRODUCTION}

Synthethic aperture radar (SAR) is the only weather and illumination independent remote sensing imaging sensor available at the moment. Several data processing techniques like Persistent Scatterer Interferometry (PSI) (Ferretti et al., 2001), SqueeSAR (Ferretti et al., 2011), and TomoSAR (Reigber and Moreira, 2000), (Zhu and Bamler, 2010), (Fornaro et al., 2015) incorporate repeated acquisitions of the same scene and thus opened up a broad range of applications. The high geometrical resolution of some decimeters combined with a temporal change detection rate of up to some $\mathrm{mm} /$ year make these techniques perfectly suitable for building monitoring tasks. Even though, the assignment of single TomoSAR points or Persistent Scatterers to buildings has been conducted already (Schunert, 2014), it however remains unclear in many cases which geometrical structure in the scene induces the coherent signal reflection. One method to increase the interpretability of particular PS is to use additional data holding different information about the sensed object. Oblique optical imagery is suitable for this purpose since it captures the earth's surface from a comparable viewing direction as SAR. Moreover, airborne oblique imagery typically offers a higher geometrical resolution than modern spaceborne SAR systems. Furthermore, the spectrum of optical sensors matches the human visual system allowing for an easy interpretation of the images. The combined use of SAR and optical imagery enables investigations about the physical nature of Persistent Scatterers and is a crucial step towards a highly precise building monitoring system.

The main contribution of this paper is to derive a mathematically proven optimal assignment of PS to their equivalents in optical oblique imagery. In order to do so, we derive a graph matching algorithm which finds the optimal relation between point features from both data types. Due to the very different appearance of the same facade in SAR and optical data, the data registration task is complicated. Therefore, we introduce some constraints about the

${ }^{*}$ Corresponding author scene structure which are justified by model knowledge about the regular alignment of facades.

In this paper we first give the context of related work followed by some fundamental considerations about the appearance of facades in SAR and optical oblique images which constitutes the motivation for the presented approach. The data registration approach is based on existing graph matching techniques. Therefore, basic concepts and particular methods of graph matching are presented in Section 3, followed by the employed method itself. The characteristics of the algorithm and its applicability is discussed with the aid of two case studies in Section 5.

\subsection{Related work}

Fusing SAR and optical nadir imagery has been conducted several times. The application context was mostly change detection. A universal approach for the registration of highly heterogeneous remote sensing data is given in (Mercier et al., 2008). A probabilistic measure based on the Kullback-Leibler-divergence of local statistics is derived under the assumption that even for very heterogeneous sensors a certain dependence between the two heterogeneous data sources exists in unchanged areas. Similar to this hypothesis we exploit the fact that regular patterns of facade objects and its inherent topology information remains constant in both mapping geometries. (Brunner et al., 2010) extract house model parameters, i.e. height, width, length, and gable roof angle, of individual buildings from an optical nadir image and use a ray tracer to simulate the corresponding SAR image. Matching this simulation with the actual SAR acquisition can answer the question whether a building has collapsed or not. This approach is limited to detached buildings. A very similar approach is given in (Sportouche et al., 2011) resulting in a threedimensional model of flat roof isolated buildings derived from a single nadir and a single high resolution spotlight TerraSAR$\mathrm{X}$ acquisition. Both approaches only regard objects on the scale of single buildings. Our approach is finer-grained and considers single PS and their actual position on the building facade which 


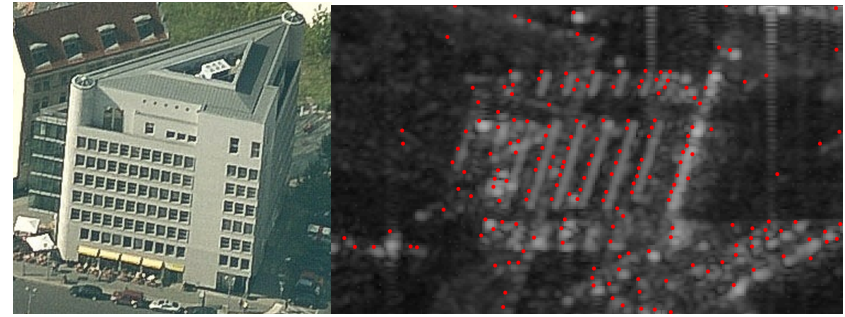

(a) optical image

(b) SAR and PS

Figure 1: One facade in (a) an optical oblique image and (b) mean amplitude SAR image with overlayed Persistent Scatteres in red. The regular pattern of windows at the facade is perceivable in both data types.

allows for high precision monitoring tasks distinguishing different parts of a building.

Facades in SAR data were also investigated in terms of approximating them as planes or curved faces (Zhu and Shahzad, 2014). The actual position of individual scatterers at the facades are neglected, however. A more detailed investigation of the scattering mechanism with the help of a precise 3D building model was conducted in (Gernhardt et al., 2015). The localization accuracy in range and azimuth of few centimeters, but some decimeters in elevation direction, could be confirmed by comparison with a precise three-dimensional building model. First attempts to exploit the regularity of PS at facades were carried out in (Schunert and Soergel, 2012) and (Schack and Soergel, 2015). Prior knowledge about the alignment of facade elements and the assumption that regular patterns of Persistent Scatterers are induced by regularly spaced objects of the same type at the facade are used to improve the elevation direction accuracy.

All above mentioned works consider the SAR data as two-dimensional images or point clouds without regarding the individual scattering mechanism. In order to study the physical nature of Persistent Scatterers (or any other three-dimensional point like features derived from a stack of SAR acquisitions) additional optical data provides valuable information which was not used as yet. Furthermore and to the best of our knowledge, a systematic exploitation of the regularity for the purpose of fusing SAR data and oblique optical imagery was not conducted up to now. This paper continues previous work on grouping Persistent Scatterers and aims at finding a correspondence in an optical oblique image (Schack and Soergel, 2015). Our goal is to enable the assignment of single PS to individual parts of the facade. In order to do so, we formulate the task as an optimization problem which we solve with a graph matching based algorithm. The registration quality is optimized comprising two terms which evaluate the matching result in terms of geometry and topology.

\section{FACADES IN SAR AND OPTICAL OBLIQUE IMAGES}

Urban areas are often characterized by multistory buildings showing regular patterns of windows or balconies at their facades. This regular appearance provides the basis for the presented registration task since it remains perceivable in both types of data. Comparing the same facade in a SAR and an optical oblique image in Figure 1 illustrates the different imaging properties of both sensors. Nevertheless, the regular alignment of windows can be seen in both data types. We use this very characteristic to establish the registration between them. The regular alignment can be ex-

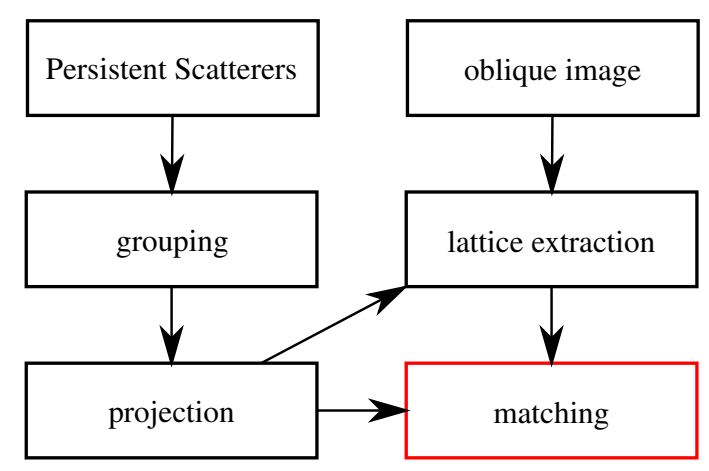

Figure 2: Flowchart of the overall processing chain. The lattice extraction in optical data is assisted by the projected grouping results of PS. The matching step is in the focus of this paper and is done in the image domain.

pressed as a lattice

$$
\begin{aligned}
& L:=\left\{a \cdot t_{1}+b \cdot t_{2}+L_{0}\right\} \\
& \text { with: }(a, b) \in \mathbb{Z},
\end{aligned}
$$

where $t_{1}$ and $t_{2}$ are the vectors describing the horizontal and vertical spacing of windows at the facade. Repeating these basis vectors $a$ and $b$ times, respectively, allows to address any point on this lattice. The offset $L_{0}$ translates the whole set $L$. For our task, $L_{0}$ can also be seen as the parameter that shifts the whole lattice toward that position in the optical image which most likely corresponds to the image of the object which induces the Persistent Scatterers. In order to find correspondences of PS in the optical image, we exploit this lattice-like regularity and conduct a search for the image correspondence that best fit the PSs.

\subsection{Data preprocessing}

The PS processing was conducted using the PSI-GENESIS processor of DLR (Adam et al., 2003) and outputs a list of PS containing the range and azimuth coordinate as well as the threedimensional geocoded object coordinates in UTM. Our method uses this set of five-dimensional PS as input for a grouping process in order to capture the regular alignment. In the data at hand, mapped by SAR systems, this regularity is preserved and leads to salient patterns in the range-azimuth plane (see Figure $1 \mathrm{~b}$ for an example). Compared to the point localization accuracy in the two-dimensional range-azimuth geometry of a few centimeters, the third coordinate of a Persistent Scatter, i.e. the elevation direction, is worse by a factor of 20 (Gernhardt, 2012). This is the reason why extracting the regular pattern is worthwhile in the range-azimuth plane. Doing so requires the segmentation of the total point cloud into subsets of PS presumably belonging to single facades. This enables the separation of scatterers of adjacent facades. The segmentation is best done in three-dimensional object coordinates. Finally, a lattice representation corresponding to (1) is fitted into the subsets. The grouping procedure itself is not within the scope of this paper and the reader is referred to (Schack and Soergel, 2014) for more details. A condensed preprocessing workflow for both data types is shown in Figure 2. A worthwhile detail is that the lattice extraction in the oblique imagery is aided by the PS grouping results.

Point like features are derived in the optical oblique imagery as matching partners for the PS. The main idea here is to exploit the facade segmentation performed within the PS point cloud to facilitate the localization of the corresponding facade in the optical image. This is done by projecting the convex hull of all three-dimensional PS into the oblique image. This transforms the 


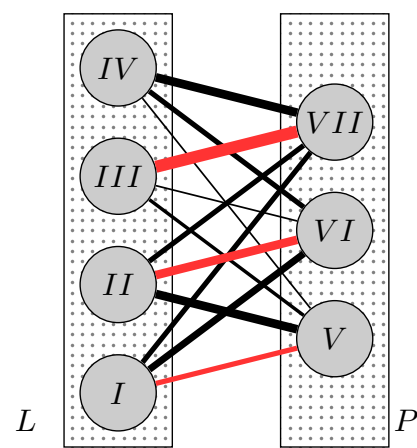

Figure 3: A bipartite graph. The set of nodes are separated into two subsets $\{L, P\}$ in a way that edges are restricted to relations between these two subsets. The edge labels $\mu$ are depicted as the thickness. A possible bipartite matching is marked in red.

problem from detecting regular facade patterns in aerial images to just extracting the regularity itself. Furthermore, the image becomes rectified in a way that the horizontal alignment of facade objects coincides with the $\mathrm{X}$-axis and the vertical alignment corresponds to the Y-axis of the image, respectively. We refer to (Schack and Soergel, 2015) where the procedure of extracting a lattice following equation 1 facilitated by PS data is described in detail.

\section{BIPARTITE GRAPH MATCHING}

A graph is a common and well established structure to describe and formalize relations between entities of any kind. In our case, nodes of the graph represent PS as well as point like features derived from the optical imagery while edges correspond to their bilateral relations. More precisely, the set of Persistent Scatterers is denoted as $P$ and the lattice points derived from the optical image as $L$, respectively. The set of edges are denoted as $E$. Every edge has a real valued number $\mu$ which attributes every relation with a score which, in a simplified view, can be seen as a measure how well the nodes adjacent to this edge could be matched. The derivation and importance of the weights $\mu$ is described in Section 4 . The bipartite graph structure at hand can compactly be written as the tuple

$$
G=(\{P, L\}, E, \mu) .
$$

Note that $P$ and $L$ are disjoint and that $E$ is restricted to edges between $P$ and $L$. Figure 3 shows an exemplary bipartite graph with 4 nodes in $L$ and 3 in $P$. The thickness of the edges between $L$ and $P$ symbolizes the edge weights $\mu$. A bipartite matching $M(G)$ is a subset of $E$ in such a way that every node in $L$ has at most one matching partner in $P$ and vice versa. Furthermore, a matching is called optimal if the solution minimizes or maximizes a certain function of the edge weights, e.g. maximizing the sum of edge weights. Such a solution is marked with red lines in Figure 3. Finding a $M(G)$ which optimizes the sum of selected $\mu$ is a standard problem in graph theory and known as linear sum assignment problems or maximum weight matching. The 'Hungarian Algorithm' solves such problems and was already developed in the 1950s (Kuhn, 1955). Several improvements in terms of efficiency were published since then ((Kao et al., 2001) and (Das and Kapoor, 2014)) and made this algorithm widely applicable to many fields where assignment problems like allocation of resources are needed. A comprehensive introduction and overview of the Hungarian Algorithm is given in (Burkard et al., 2009). For our purposes it is sufficient to summarize the algorithm and its important characteristics as follows:
Hungarian Algorithm: Suppose a distance matrix $D$ with dimension $|P| \times|L|$ is given where $|P|$ denotes the number of PS and $|L|$ the number of lattice nodes derived from the optical image, respectively. The entries in $D$ are positive and real valued. The Hungarian Algorithm returns those matrix elements in $D$ which maximize the sum of selected elements (optimality) under the constraint that as many as possible entries are selected preserving a bipartite matching (maximality).

The entries in $D$ can be seen as the $\mu$ weights in the tuple representation (2) of the graph. For the remainder of this paper the graph structure and the matrix representation are used interchangeably. Preserving a bipartite matching can be seen as the constraint that every column and row is allowed to contain at most one selected entry. In general the amount of PS is lower than the number of lattice nodes derived from optical imagery. In such cases only $|P|$ lattice nodes in $L$ get matched to a PS. In the following section we derive an iterative procedure which applies the Hungarian Algorithm given a distance matrix $D$ under some topology constraints for $P$, namely incorporating the PS grouping result.

\section{METHOD}

As outlined in Section 2 the direct projection of PS into oblique imagery is error-prone due to the inaccurate elevation coordinates of the PS. Another source of uncertainty is the orientation of the optical camera. In order to make the matching more robust against such uncertainties, we exploit the regular structure of the facades under investigation. The key idea is to condense this prior knowledge about the regularity and the fact that the neighboring properties, i.e. the topology of facade objects, are preserved in both imaging geometries. The implementation of these considerations is done by minimizing a distance matrix consisting of two terms: First, a geometrical distance in image coordinates between matched PS and optical lattice nodes has to be small. This term is denoted by $D_{\text {geom }}$. Second, the topology of the PS should be preserved when projected into the optical image. The term capturing this property is denoted by $D_{\text {topo }}$. Both terms can be adjusted in terms of how much they contribute to the overall solution. This leads to the joined distance matrix

$$
D=\alpha D_{\text {geom }}+(1-\alpha) D_{\text {topo }} .
$$

The weighting parameter $\alpha$ controls the contribution of each term and is defined between 0 and 1 . A value of $\alpha=1$ degenerates the solution to a simple geometrical problem while $\alpha=0$ means totally neglecting the geometrical information and only considering the topology. How both terms are derived and a short discussion of them are given in the following two sections.

\subsection{Geometrical information}

The first term in equation (3) captures the geometrical consistency of a matching. The highly anisotropic error budget of threedimensional PS coordinates due to the large error in elevation direction has to be taken into account. Matching is established in the image coordinate system which requires projecting the PS. The covariance matrix of the PS in image coordinates $C$ can be derived by error propagation from object coordinates and a-priori information about the orientation parameters. $D_{\text {geom }}$ is a matrix with one column for every PS and one row for every lattice node. The elements are the Mahalanobis distances between every PS and image lattice node:

$$
D_{\text {geom }}(i, j)=\sqrt{\left(L_{i}-P_{j}\right)^{\top} C\left(L_{i}-P_{j}\right)},
$$


where $L_{i}$ denotes the $i$ th lattice node and $P_{j}$ the $j$ th PS projected into the image.

\subsection{Topology information}

Analogously to the lattice representation of grouped PS following Equation (1), the regular structure of facade buildings present in optical images can also be described by two spanning vectors (or spacings in the case of rectified images). For a better separation between a lattice grouped in the optical image and a product of the PS-grouping we denote the lattice coordinates for the first one with $a$ and $b$, while for the latter $u$ and $v$ are used. Then, a single node of this regularity can be represented as its two integer repetition $u, v$ of these spacings. Projecting all PS into the optical image and matching them one-to-one to optical lattice nodes yields the following information: Every PS now has an index $(a, b)$ as well as an index $(u, v)$ of the matched lattice node. Figure 4 shows a simple example of such an intermediate matching result. The regular lattice nodes derived from the optical image are marked with black points while the projected PS are depicted as red crosses. Their $(a, b)$ indices are written in red directly next to the PS while the $(u, v)$ coordinates are given via the axes. The grouping result of the PS in the SAR domain is shown as red solid lines. A matching between them and the optical lattice is symbolized as blue dashed lines. The topology is consistent except for the two PS $(a=2, b=1, u=6, v=10)$ and $(a=2, b=2, u=6, v=9)$. Modifying the matching for these two PS would harmonize the topology but increase the geometrical distance of the matching. Comparing this example with the problem statement in Section 4, Figure 4 can be interpreted as follows: In terms of the tradeoff between geometrical and topological consistency the sum of the length of the blue dashed lines are a measure of the geometrical quality while the topological consistency is captured by comparing the indices.

Analogously to the geometrical measure, $D_{\text {topo }}$ is a matrix with one column for every PS and one row for every lattice node. The elements are computed as

$$
D_{\text {topo }}(i, j)= \begin{cases}0, & \text { if } P_{j}(a)=L_{i}(u) \wedge P_{j}(b)=L_{i}(v) \\ 1, & \text { otherwise }\end{cases}
$$

This means that $D_{\text {topo }}$ has zero entries wherever the topology of the matched lattice nodes is consistent with the topology of the corresponding PS in the SAR geometry and 1 otherwise. This model is rather simple but captures well the prior knowledge about the topology and empirically leads to satisfactory results. An important consideration is neglected in the described example and Figure 4: The offset between the $(a, b)$ and $(u, v)$ indices has to be considered. This is done by performing a majority voting scheme over the differences $a-u$ and $b-v$, respectively, assuming that the projection of the majority of PS into the optical image is correct in terms of topology. This assumption was met in all empirical investigations done so far.

The mutual dependence of $D_{\text {topo }}$ and $D_{\text {geom }}$ via an iterative procedure is an important element of the overall approach and the key element for the derived Iterative Bipartite Matching algorithm.

\subsection{Iterative Bipartite Matching}

Given a set of Persistent Scatterers originating from a single facade and an optical oblique image from the same building face, we aim at finding two things: First, a matching $M$ between the PS and features derived from the optical image as well as their

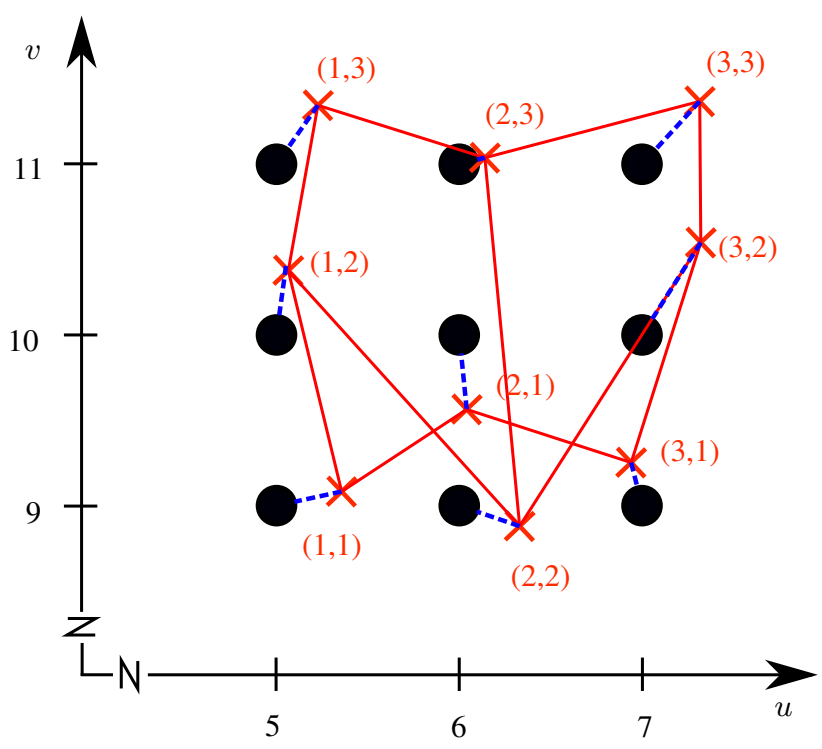

Figure 4: Example of an intermediate matching result. Black dots mark lattice nodes which were derived from the rectified optical oblique image. They can be described by their $(u, v)$ indices. Red crosses symbolize PS projected into the optical image. Their indices $(a, b)$ which are the result of the grouping procedure in the SAR domain are given in red. The matching result of this iteration is shown as blue dashed lines. A contradiction is present in the lower middle part.

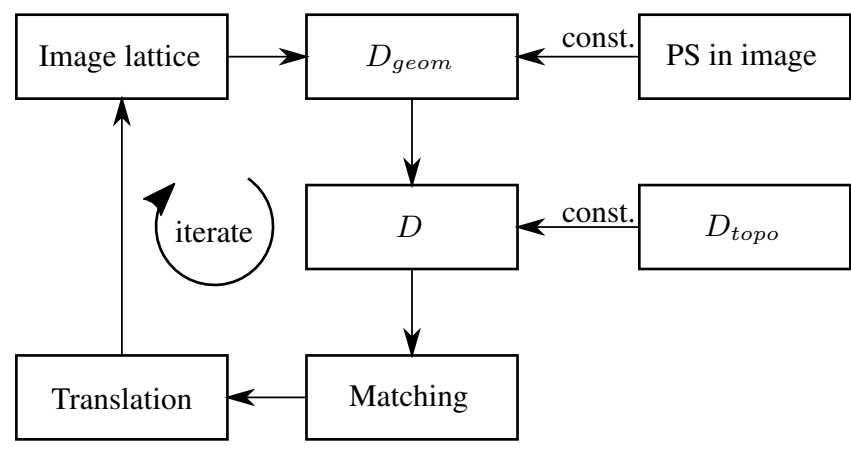

Figure 5: Flow chart of the iterative bipartite matching procedure. The PS in image coordinates as well as the topological information $D_{\text {topo }}$ are given and constant during all iterations. The distance matrix $D$ is composed of the geometrical and topological distance matrices. $D$ is subject to a standard bipartite matching approach which leads to changed image coordinates of the lattice nodes. 
actual position $L$ in the image. Since we exploit the regular alignment of window elements, all matching partners in the image can be described as the set of nodes defined by a lattice as given in Equation (1). The spacings in horizontal and vertical direction in the rectified image are not part of the optimization procedure and thus, the position is unambiguously determined by the lattice offset $L_{0}$. A matching $M(D)$ can be found for a given distance matrix $D$ which is built according to Equation (3). These considerations can be put into an analytical form and let us formulate the following minimization problem:

$$
\begin{aligned}
\underset{M, L_{0}}{\arg \min } \sum_{m}^{M(D)} \sqrt{\left(L_{m}-P_{m}\right)^{\top} C\left(L_{m}-P_{m}\right)} \\
\text { w.r.t. } D=\alpha D_{\text {geom }}\left(L_{0}\right)+(1-\alpha) D_{\text {topo }},
\end{aligned}
$$

where $m$ is the index over all matched pairs of PS and corresponding lattice nodes. An important observation concerning the solution of the above problem is that both parameters to optimize are mutually dependent: Changing the lattice shift $L_{0}$ may lead to a different matching $M$ and vice versa. In order to cope with this mutual dependency, we derive an iterative approach of which the main idea is inspired by the expectation maximization algorithm (Dempster et al., 1977): Fixing the matching and optimizing the shift followed by an updated matching iteratively converges under certain criteria which are stated below.

A flowchart of the optimization scheme is given in Figure 5. The position of the PS in the image as well as the topology are constant. The iterative procedure starts with calculating the geometrical distance between all PS and image lattices considering the anisotropic error metric. Prior knowledge about the topology in SAR geometry is introduced into this matrix by considering the weighted distance matrix $D$ following Equation (3). Performing a standard bipartite matching results in a matching $M(D)$. Based on this matching the shift $T$ is computed which minimizes the sum of distances of all matched pairs. With these new lattice positions, the distance matrix of the next iteration is created and the whole procedure is repeated until the solution converges. Algorithm 1 states the described procedure in four steps and is the basis for the following description, which explains why the presented approach is optimal and guarantees convergence.

Data: Image coordinates of PS; topology matrix $D_{\text {topo }}$

Result: Assignment $M$ of PS to lattice nodes; image coordinates of lattice origin $L_{0}$

Step 0:

Initialize lattice origin $L_{0}$

Initialize shift $T=(0,0)$

while solution has not converged do

Step 1:

Apply shift: $L_{0} \leftarrow L_{0}+T$

Step 2:

$D \leftarrow \alpha D_{\text {geom }}\left(L_{0}\right)+(1-\alpha) D_{\text {topo }}$

Step 3:

$M \leftarrow$ Hungarian algorithm $(D)$

Step 4:

$T \leftarrow$ average $\operatorname{shift}(M)$

end

\section{Algorithm 1: Iterative Bipartite Matching}

The proof of optimality and convergence is based on two basic theorems. The first simply states that step 1 minimizes the current solution for a given matching $M(D)$. Note that the averaging itself is done in step 4 but directly followed by step 1 in the next iteration.

Theorem 1 (Optimal shift). Given a bipartite matching with $|M|$ matched pairs in a Mahalanobis distance metric, averaging the shift between matched nodes minimizes the sum of the Mahalanobis distances over all matches in $M$.

Proof. The sum of $|M|$ Mahalanobis distances is

$$
f: \sum_{m=1}^{|M|} \sqrt{\left(d_{m}-t\right)^{T} C\left(d_{m}-t\right)},
$$

where $d_{m}$ are the distances between matched pairs and $t$ is the global shift. The shift which minimizes $f$ can be found by finding the minimum of the derivative of $f$ with respect to $t$. Since the square root increases strictly monotonically it can be ignored for finding the minimum. Thus,

$$
\frac{\partial f}{\partial t}: \frac{\sum_{m=1}^{|M|} C d_{m}}{\sum_{m=1}^{|M|} C}=\frac{1}{|M|} \sum_{m=1}^{|M|} d_{m} .
$$

Step 2 updates the distance matrix $D$. It is important to note, that $D_{\text {topo }}$ as well as $\alpha$ are constant throughout all iterations and, thus, $D$ only depends on $D_{\text {geom }}$. The Hungarian Algorithm always returns the optimal solution for a given distance matrix. Therefore, changing the elements in $D_{\text {geom }}$ in the way as described above leads to Theorem 2 .

Theorem 2 (Updated matching). An optimal matching $M_{1}$ with matching costs $c_{1}$ in a bipartite graph $G=(\{P, L\}, E, \mu)$ is given. If the translation which minimizes the sum of the distances is applied on one set of the bipartite graph, i.e. L, computing again an optimal matching will always yield a matching $M_{2}$ with matching costs $c_{2}$ in a way that $c_{2} \leq c_{1}$.

Proof. Let $T$ be the averaged shift minimizing the sum of Mahalanobis distances. Applying this shift to $L$ but holding on to the matching $M_{1}$ leads to matching costs which are at most as high as $c_{1}$ according to Theorem 1. Performing the optimal matching on the shifted nodes will always yield smaller or equally high matching costs as $c_{1}$ since otherwise the matching would not be optimal.

Thus, every iteration leads to matching costs smaller than the previous iteration. The algorithm always converges since the matching costs decrease or stay constant from iteration to iteration. A configuration where the algorithm jumps between two equal states is conceivable but did not occur during our empirical investigations. The parameter $\alpha$ plays an important role in terms of balancing the two mutually dependent impacts for the overall solution. The geometry and the topology information favour different matchings. Neglecting the topology information altogether, i.e. $\alpha=1$, leads to a bipartite matching which minimizes the geometrical distance between the lattice points and the PS projected in the image and can be interpreted as an 'Iterative closest point'-like procedure. On the other hand, neglecting the geometry information and just considering the topology information, i.e. $\alpha=0$, leads to a solution in total accordance with the topology derived from the SAR grouping step. In summary, either the influence of projection errors due to a defective outer orientation, for instance, is dominant in the geometry-only solution, or the position of the lattice in the image has no influence at all in the topology-only solution. This, and other important aspects of 


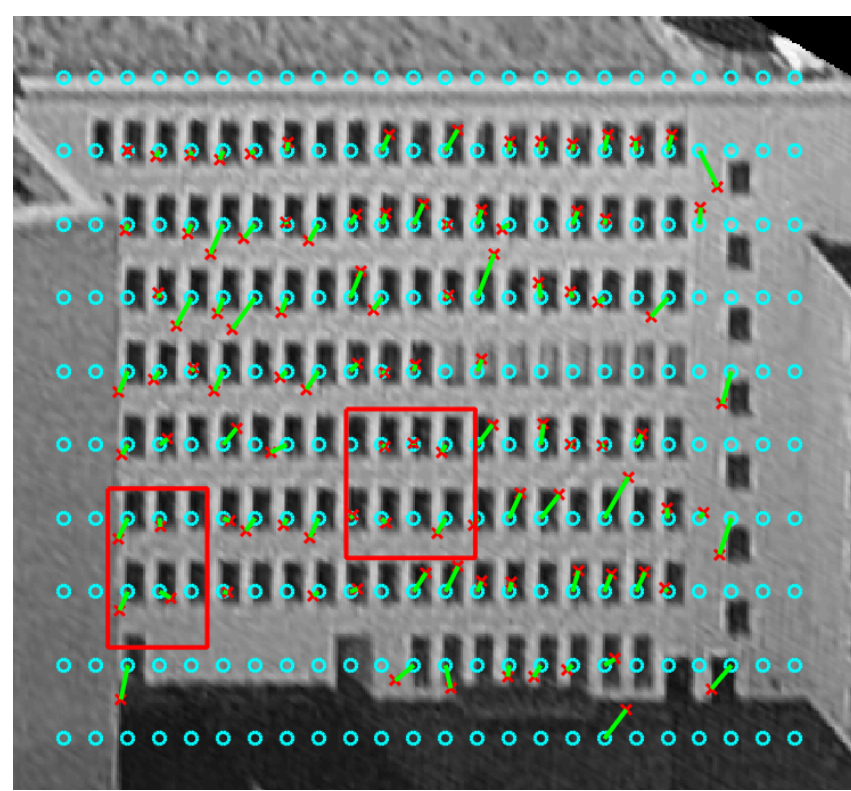

Figure 6: Overview of the facade 'Lindenstraße'. Red crosses: Persistent Scatterers projected into the image. Cyan circles: Final lattice nodes after convergence. Green lines: matching vectors between PS and corresponding lattice nodes. Two areas marked with red rectangles are used for further investigations.

the presented method are empirically shown in the following two case studies.

\section{CASE STUDIES}

Two case studies are presented to show the applicability of the presented method and to discuss some characteristics of the algorithm. We use the following data captured from the city center of Berlin, Germany.

- A Persistent Scatterer point cloud derived from 54 TerraSAR$\mathrm{X}$ High Resolution Spotlight acquisitions with an incidence angle of $39^{\circ}$. The spatial resolution in range and azimuth direction is approximately $60 \mathrm{~cm}$.

- The imagery consists of several $45^{\circ}$-obliques captured during two campaigns in 2006 and 2010. The ground sampling distance in the scene center lies in between $10 \mathrm{~cm}$ to $15 \mathrm{~cm}$.

\subsection{Case study Lindenstraße}

The influence of the parameter $\alpha$ is discussed with the aid of this case study. The facade under investigation belongs to a seven storey building in the city centre of Berlin. An overview including a matching result for $\alpha=0.5$ is given in Figure 6. The PS projected into the oblique image are marked with red crosses. The matching to the lattice nodes (cyan circles) are given as green lines. Two issues are noticeable: First, the overall consistency between PS positions and lattice nodes seems to be meaningful. The optimization procedure yielded the lower left corner as the optimal matching partner for the PS. Second, the directions of the matching vectors (green lines) are not randomly distributed but are very similar which coincides with the projection of the elevation direction into the image.

The algorithm is run for the same facade but varying values of $\alpha$. Figure 7 shows the geometry and topology scores as well as the

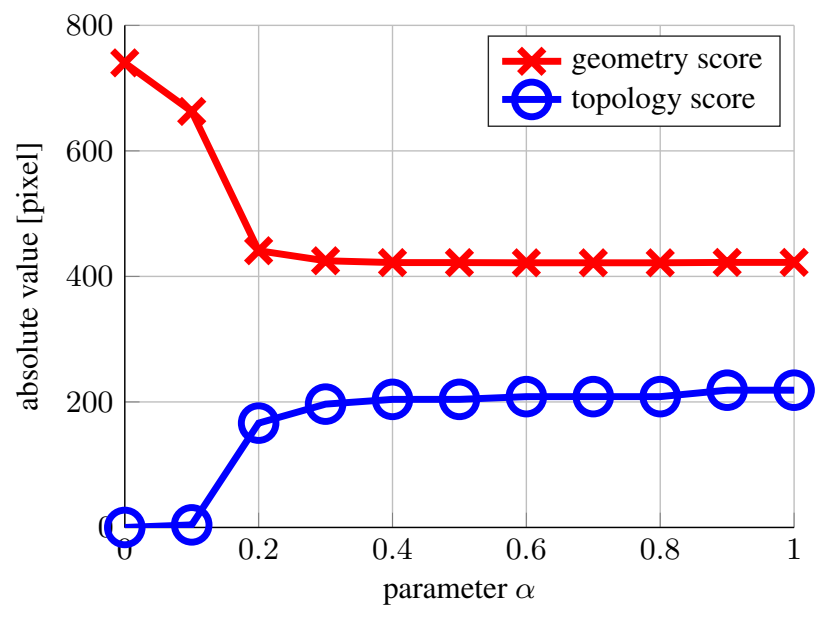

(a) Dependence of the geometry and topology score on $\alpha$. Beginning from $\alpha=0.3$ no distinct changes for higher values of $\alpha$ can be recognized.

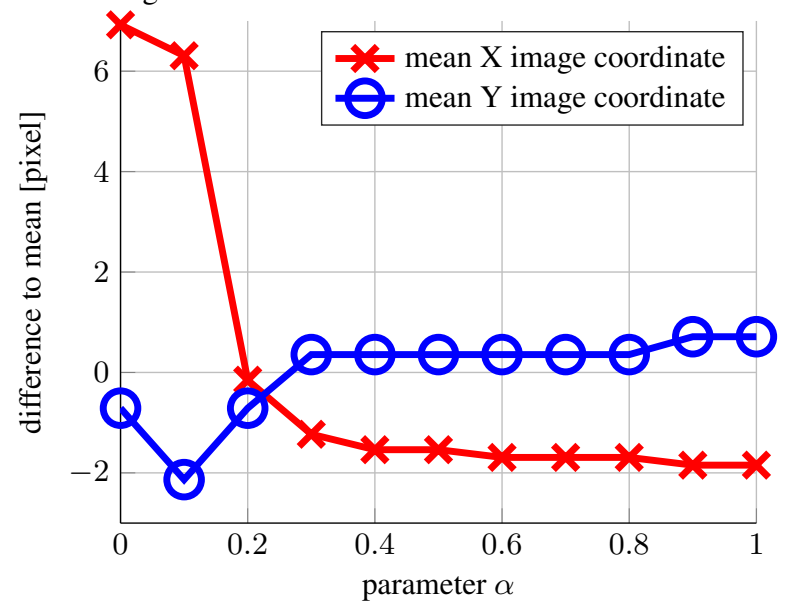

(b) Dependence of the mean $\mathrm{X}$ and $\mathrm{Y}$ coordinates of the lattice node positions in image coordinates relatively to the mean. Four different states can be discerned.

Figure 7: Dependence of geometry and topology scores (a) as well as the $\mathrm{X}$ and $\mathrm{Y}$ coordinates (b) of the final lattice nodes on parameter $\alpha$ 


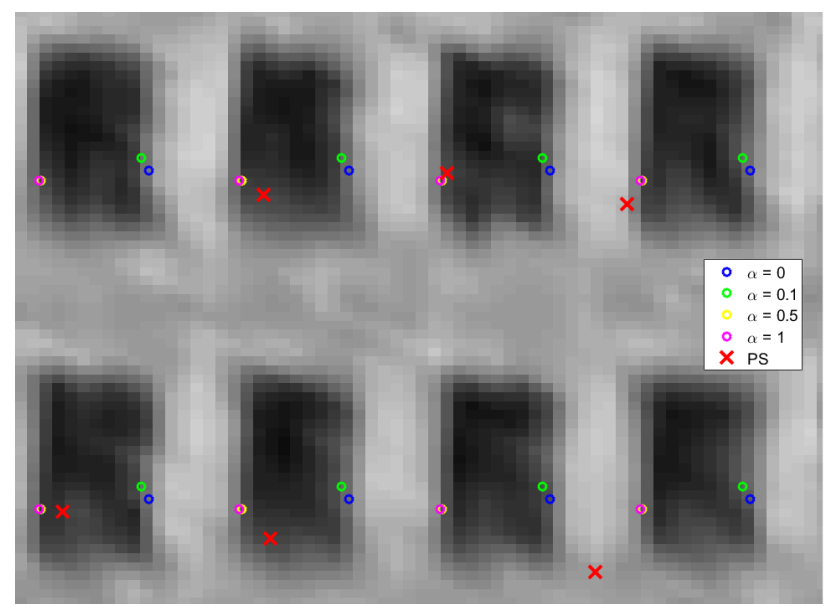

Figure 8: Different positions of lattice points as a function of the parameter $\alpha$. Background: cut out grayvalue image of the facade. The original PS positions are marked as red crosses. The resulting lattice node positions which are dependent on the parameter $\alpha$ are marked as colored circles.

final position of the lattice nodes as a function of $\alpha$. The geometry score in Figure 7a is simply the summed length of matching vectors for the final solution in pixels, while the topology is evaluated by measuring the sum of distances of matches with nonconsistent topology. Setting $\alpha=0$ leads to a solution which is solely governed by the topology. Thus, the corresponding score is 0 while the geometry penalty is at its maximum. Increasing $\alpha$ means putting more emphasis on the geometry, lowers the corresponding score but consequently increases the topology score. This behaviour can be recognized until a value of $\alpha=0.3$. Strikingly, higher values do not change the scores any further. The dependence of $\mathrm{X}$ and $\mathrm{Y}$ coordinates from $\alpha$ shows a similar behaviour. Figure $7 \mathrm{~b}$ shows the change of the centroid of all lattice nodes dependent on $\alpha$. Since one of our two aims is to find the most likely image coordinates of the matching partners for all PS, it is worthwhile noticing that the algorithm is stable with respect to $\alpha$. Only four different states can be identified if all differences below one pixel per coordinate are assumed to represent equal results.

An important question is how these four configurations differ from each other in terms of the matching and the positions of the matched lattice nodes in the image. Based on the previous findings, Figure 8 shows the final results for four different $\alpha$ values. The depicted scene is a cut out from Figure 6, marked by the right red rectangle. For low $\alpha$ values, i.e. $\alpha=0$ and $\alpha=0.1$, the lattice nodes tend to be on the right side of the windows. For $\alpha=0.5$ and $\alpha=1$ the lattice nodes lie on the lower left corner of the windows. Considering the SAR sensing geometry and the orientation of the facade with respect to the sensor line of sight, this left corner is assumed to be the correct side. Since $\alpha=1$ means totally neglecting the topology information, the finding prompts the question why this sort of information is helpful at all. Figure 9 gives the answer. The depicted scene is a cut out from Figure 6, marked by the left red rectangle. Relying only on the geometry information, see Figure 9b, causes the lower right PS (yellow circle) to get assigned to a lattice node which violates the topology gained by the PS grouping. Incorporating this information offers a more realistic result, as shown in Figure 9a.

\subsection{Case study Charlottenstrasse}

A second case study is presented in Figure 10 in order to show the robustness of the approach. The positions of the projected

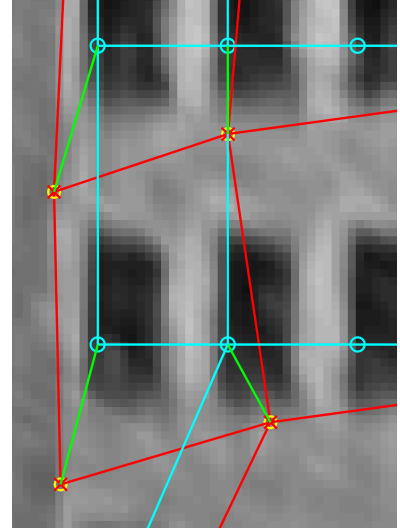

(a) $\alpha=0.5$

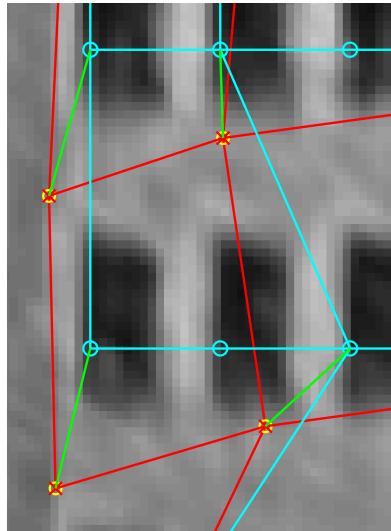

(b) $\alpha=1$
Figure 9: Cut out detail of Figure 6. Influence of topology for (a) $\alpha=0.5$ and (b) $\alpha=1$. Red lines: grouping result of PS in rangeazimuth geometry, projected into the image. Green lines: Matching vectors between PS and lattice nodes. Cyan lines: topology of matched lattice nodes. Topology is consistent in (a) but not in (b).

Persistent Scatterers are marked with red crosses while the final lattice nodes are given as cyan circles. The matching vectors are depicted in green. Even though the facade is partly covered by a different building in the optical image and some PS at the upper region are induced by objects not being part of the regularity, the matching is meaningful. The lower left window corner coincides with considerations about the SAR sensing geometry and the facade orientation with respect to the sensor line of sight.

\section{CONCLUSION AND OUTLOOK}

We derived an iterative graph matching algorithm in order to assign lattice nodes derived from optical oblique imagery to Persistent Scatterers at facades. The presented approach is proved to converge and to yield the optimal solution in terms of minimizing the Mahalanobis distance between the projected PS in the image and the optical lattice nodes.

At the moment, the approach is limited to building faces with regularly aligned facade elements. We exploit this pattern structure to establish the connection between point like features from very different sensors allowing for a better interpretation of the mechanisms inducing Persistent Scatterers. The matching procedure itself, however, does not rely on any regular alignment of objects to match. Therefore, an extension to more general facades is part of upcoming research. Furthermore, it is planned to enlarge the extension from single facades to investigation areas of some square kilometers in extent and to apply the approach to change detection tasks. Further work will also investigate more complex approaches for the binary model (5) capturing the topology information. One possibility is to weight the entries of $D_{\text {topo }}$ dependent on how much the indices differ from each other.

\section{REFERENCES}

Adam, N., Kampes, B., Eineder, M., Worawattanamateekulb, J. and Kircher, M., 2003. The development of a scientific permanent scatterer system. In: Proceedings of ISPRS Workshop High Resolution Mapping from Space.

Brunner, D., Lemoine, G. and Bruzzone, L., 2010. Earthquake damage assessment of buildings using VHR optical and SAR imagery. IEEE Transactions on Geoscience and Remote Sensing 48(5), pp. 2403-2420. 


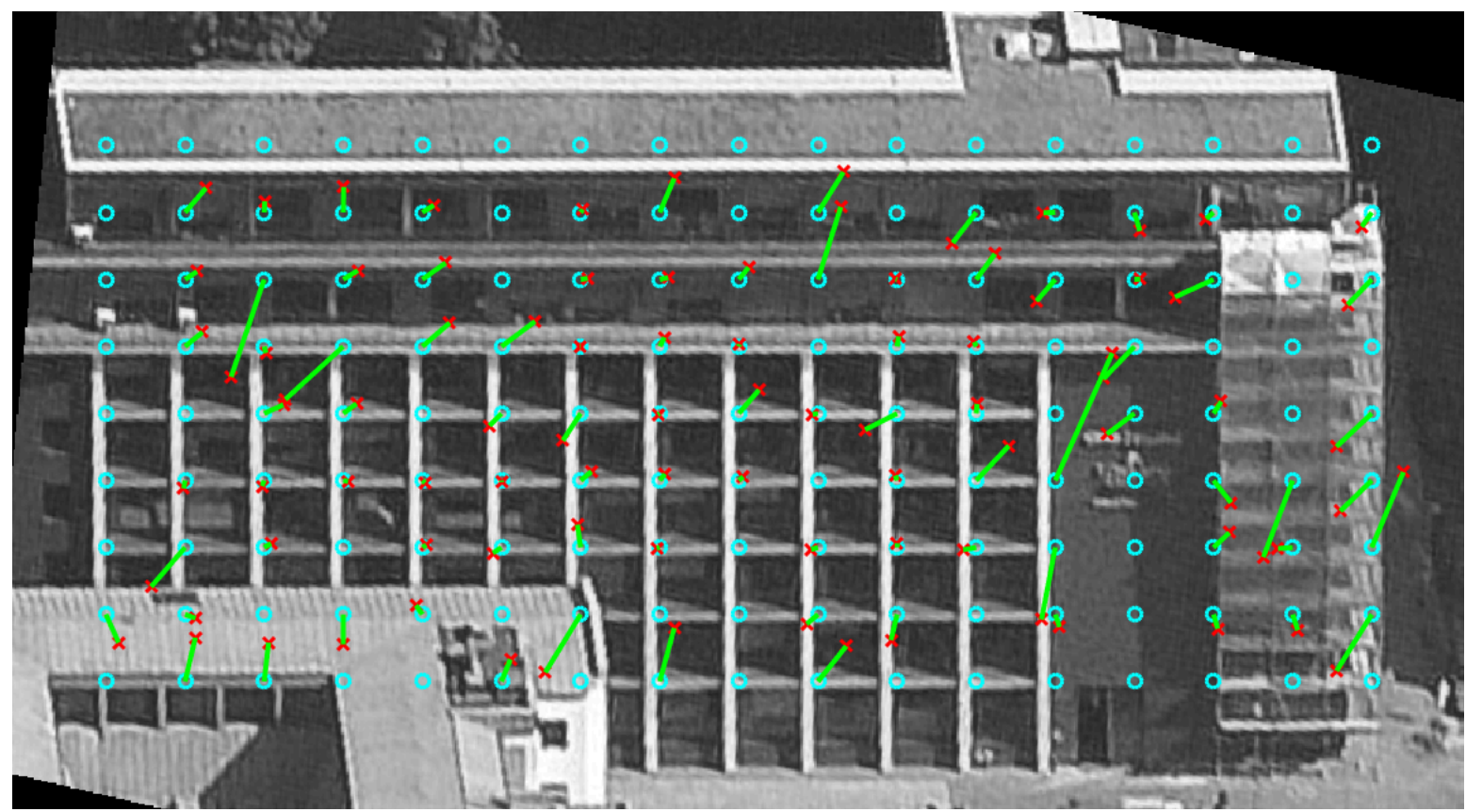

Figure 10: Matching result for case study 'Charlottenstrasse' with $\alpha=0.5$. Original PS positions are marked as red crosses. Lattice nodes are drawn as cyan circles. The matching is indicated by green lines.

Burkard, R., Dell'Amico, M. and Martello, S., 2009. Assignment Problems. Society for Industrial and Applied Mathematics, Philadelphia.

Das, S. and Kapoor, K., 2014. Fine-Tuning Decomposition Theorem for Maximum Weight Bipartite Matching. In: T. Gopal, M. Agrawal, A. Li and S. Cooper (eds), Theory and Applications of Models of Computation, Springer International Publishing, pp. 312-322.

Dempster, A. P., Laird, N. M. and Ruby, D. B., 1977. Maximum Likelihood from Incomplete Data via the EM Algorithm. Journal of the Royal Statistical Society. Series B 39(1), pp. 1-38.

Ferretti, A., Fumagalli, A., Novali, F., Prati, C., Rocca, F. and Rucci, A., 2011. A new algorithm for processing interferometric data-stacks: SqueeSAR. IEEE Transactions on Geoscience and Remote Sensing 49(9), pp. 3460-3470.

Ferretti, A., Prati, C. and Rocca, F., 2001. Permanent Scatterers in SAR interferometry. IEEE Transactions on Geoscience and Remote Sensing 39(1), pp. 8-20.

Fornaro, G., Verde, S., Reale, D. and Pauciullo, A., 2015. CAESAR: An Approach Based on Covariance Matrix Decomposition to Improve Multibaseline-Multitemporal Interferometric SAR Processing. IEEE Transactions on Geoscience and Remote Sensing 53(4), pp. 2050-2065.

Gernhardt, S., Auer, S. and Eder, K., 2015. Persistent scatterers at building facades evaluation of appearance and localization accuracy. ISPRS Journal of Photogrammetry and Remote Sensing 100 , pp. $92-105$.

Gernhardt, S. M., 2012. High Precision 3D Localization and Motion Analysis of Persistent Scatterers using Meter-Resolution Radar Satellite Data. PhD thesis, Technical University Munich. Published in: DGK Reihe C, Verlag der Bayerischen Akademie der Wissenschaften, in Kommission beim Verlag C.H. Beck, München.

Kao, M.-Y., Lam, T.-W., Sung, W.-K. and Ting, H.-F., 2001. A Decomposition Theorem for Maximum Weight Bipartite Matchings. SIAM Journal on Computing 31(1), pp. 18 - 26.
Kuhn, H. W., 1955. The Hungarian method for the assignment problem. Naval research logistics quarterly 2, pp. 83-97.

Mercier, G., Moser, G. and Serpico, S. B., 2008. Conditional copulas for change detection in heterogeneous remote sensing images. IEEE Transactions on Geoscience and Remote Sensing 46(5), pp. 1428-1441.

Reigber, A. and Moreira, A., 2000. First demonstration of airborne SAR tomography using multibaseline L-band data, IEEE Trans. IEEE Transactions on Geoscience and Remote Sensing 38(5), pp. 2142-2152.

Schack, L. and Soergel, U., 2014. Exploiting Regular Patterns to Group Persistent Scatterers in Urban Areas. IEEE Journal of Selected Topics in Applied Earth Observations and Remote Sensing 7(10), pp. 4177-4183.

Schack, L. and Soergel, U., 2015. Matching Persistent Scatterers to Optical Oblique Images. In: IEEE Conference on Computer Vision and Pattern Recognition Workshops (CVPRW), 2015, IEEE, Boston, MA, USA, pp. 52-60.

Schunert, A., 2014. Assignment of Persistent Scatterers to Buildings. PhD thesis, Leibniz Universität Hannover. Published in: DGK Reihe C, Verlag der Bayerischen Akademie der Wissenschaften, in Kommission beim Verlag C.H. Beck, München.

Schunert, A. and Soergel, U., 2012. Grouping of Persistent Scatterers in high-resolution SAR data of urban scenes. ISPRS Journal of Photogrammetry and Remote Sensing 73, pp. 80-88.

Sportouche, H., Tupin, F. and Denise, L., 2011. Extraction and three-dimensional reconstruction of isolated buildings in urban scenes from high-resolution optical and sar spaceborne images. IEEE Transactions on Geoscience and Remote Sensing 49(10), pp. 3932-3946.

Zhu, X. X. and Bamler, R., 2010. Very high resolution spaceborne SAR tomography in urban environment. IEEE Transactions on Geoscience and Remote Sensing 48(12), pp. 4296-4308.

Zhu, X. X. and Shahzad, M., 2014. Facade reconstruction using multiview spaceborne TomoSAR point clouds. IEEE Transactions on Geoscience and Remote Sensing 52(6), pp. 3541-3552. 